\title{
A web tool to linearize knowledge search
}

\author{
Yoshida Rao ${ }^{1}$ \\ IIT Madras, Chennai, India
}

\begin{abstract}
In Social Network analysis (SNA), we have a structure called Graphs, which has nodes and lines connecting those nodes. These lines are called edges. There is a metric in Graphs called distance. In an ideal Graph in SNA, each node should be connected to all of the other nodes, that is distance should be one. In this paper we are suggesting a Graph of scientific terminologies. There is a content corresponding to each scientific terminology and that content has tags (keywords). So the distance of the terminology to the tags of its content is one. Now those tags have their own content, and that content has more tags. So we have a Graph of terminologies. What we suggest is that as we build up the content in the website, we try to reduce the distance between the tags. This process in Graph terminology is called completing the Graph.

So we are suggesting a Content Management System, where we want to build up quality scientific content. Apart from reducing the distance, we propose a method of making a linear list of tags, which linearizes the task of literature search. We discuss in the paper the advantages of the linearizing process.

Keywords: Social Network Analysis, Education Technology, Web Product, Content Management System
\end{abstract}

\section{Introduction}

Information. There is an increasing cascading of knowledge in books and the internet, such that a student or researcher finds it difficult to gather knowledge

\footnotetext{
1yoshidarao5@gmail.com https://www.linkingresearchlogic.com/
} 
in a finite time and in a manner that he/she can remember it and use it suitably.

Wherever students see there is a spider web of knowledge, it would be much easier if knowledge could be presented in a linear list. For example, if one reads an article on Wikipedia then that article is having links to a lot many articles. In fact in a spider web form each article is in some manner connected to all the articles.

So if one wants to thoroughly go through an article one has to study all the links also, and moreover those link items will have more links.

We have devised a methodology, to create a web tool to help the student in this literature search.

\section{Methodology}

Method. When a student or researcher reads a topic, there are various terminologies (key words/short word terms) involved. He/she searches those terms and finds more content. But that content is having more terms. Many students also want such books which they can read cover to cover. But seldom there exists such books in higher studies. The student is left with no option but to combine the knowledge from various sources. Here comes our web tool to student's help. Student reads one content, and writes down a brief summary of it, and then he/she writes the terms in it as tags. He puts the tags in a list called "tags". These tags are simultaneously automatically entered into a list called "questions". The questions which have not been answered in the form of 25 content, are placed in an "unanswered questions" list. So there are three lists Tags, Questions, Unanswered questions. What students are required is to keep adding content items on the topics in "unanswered questions" list. So the task becomes to search and answer questions in one linear list. This type of list has many advantages as discussed in the paper below. 


\section{Background of Knowledge Graphs}

Knowledge Graphs. In Social Network Analysis (SNA) graphs play a central role. [ $\mathbb{Z}, \mathbf{2}, \mathbf{3}]$ Graphs are nodes connected by lines called edges. In SNA the importance of a node is not by what node does, but by how many nodes it connects. If these nodes are taken as people then the importance of a person is not by what it does, but to how many other nodes (people) it connects to. (This is not generally true, because what a person does is also important). But if we

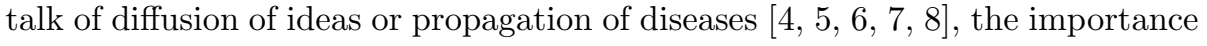
is actually by how many people one person connects to.

Now comes another issue of SNA of ideas, where nodes are ideas with lines (edges) connecting them. The brain might be taken to be working in this manner. For the brain, ideas which connect to more ideas are important. When a new idea comes, the brain tries to connect that idea to already existing ideas. If the new idea is able to connect to more ideas it is retained by the brain, otherwise it is forgotten. So the brain can be taken as a graph of ideas with ideas

45 as nodes and lines (edges) connecting them. The brain is truly a SNA of ideas, as it attaches very less importance to individual ideas but more importance to how many ideas that particular idea connects to.

\section{Our Suggestions}

\section{Suggested Points.}

1. Our system is very simplified.

2. It doesnt use AI or ML. So students can straightforwardly understand which content item is connected to which item and for what reason.

3. This is a relatively cheap project. The cost of this project is around three thousand dollars which is much less compared to other such projects in the market.

4. Since this project does not use AI or ML, students can easily appreciate the working of the project. 
5. Students can purchase a version of a project for 100 dollars with 1000 basic contents in Physics or Chemistry, and then can build on it with their own content.

6. If a student doesnt want to purchase an independent copy of the project, they can browse the content of this project for free.

7. If a student wants to submit a content of 80 words, then he can get paid 1 dollar.

8. So this project opens up the possibility of earning more money for students.

9. This project provides a cheap learning opportunity for K12 students initially.

10. This project provides a bound on the number of content items required for a course work in a particular field. This project provides an add to cart button by which a person surfing the website can select the contents of his/her area of interest. This is like pins on the pinterest website.

\section{Demonstration of the methodology}

Methodology Demonstration. We have taken examples of Physics and Chemistry

to demonstrate the product. Fig $⿴ 囗$ I shows content for Speed in Physics. Fig $\square$ shows the tags and questions raised by this content: Newtons First law and Velocity. Same way Fig $\mathbf{B}$ and Fig $\mathbf{Q}$ shows the entry for content for Light.

Fig 5 and Fig 6 show how these tags are entered in the backend of the product.

Fig $\square$ and Fig $\square$ give the all questions and unanswered questions lists.

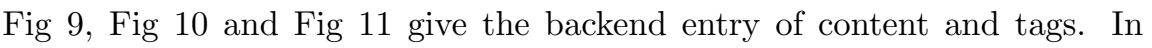
the tags entry there is a Question Answered field where the question which the current content is answering is entered. After this the question is removed from the Unanswered questions list. So as more contents are entered unanswered questions list becomes smaller. 


\section{Questions Answered : Speed}

Cause Statement : Given a physical quantity called Speed.

Effect Statement : (1) It is the distance travelled by an object per unit of time. (2) It is a scalar quantity as it only depends on magnitude and has no direction. (3) In this context it is quite different from velocity, however velocity depends on the direction of the movement. (4) The SI Unit of Speed is $\mathrm{m} / \mathrm{s}$. (5) Speed is calculated as distance upon time as: Speed =distance /time.

\section{Summary Reference Tags Question Raised}

Add to Cart

Figure 1: Student in Physics starts with the definition of speed. So he enters the content for it. This is the front end display of the content. Backend data entry would be shown in later figures.

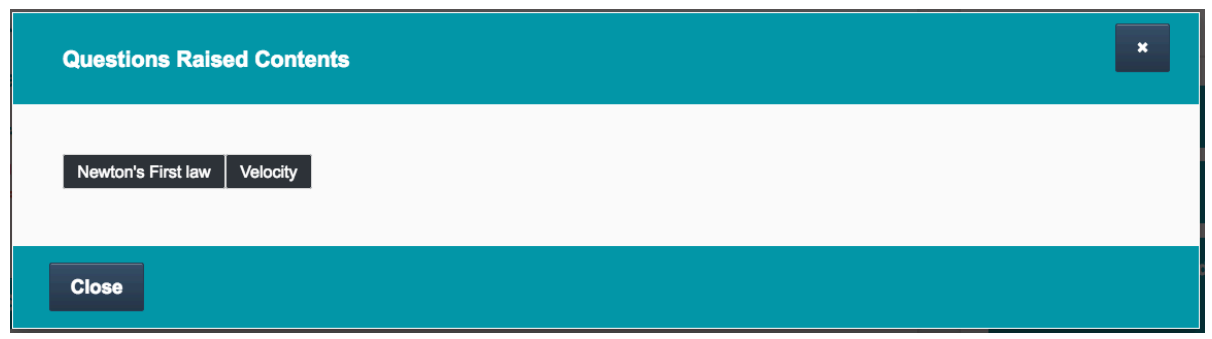

Figure 2: These are the tags with speed: Newtons First law and Velocity.

The advantage to the student is that in his/her knowledge search, he/she has to deal with a linear list of unanswered questions.

प

\section{Current Work}

Current Work. The content for a tag (keyword or terminology) is displayed. Also displayed are the tags that are at one distance from the tag. We feed these node and edges data into a Graph Analysis Software [9]. And we find tags that are at a distance two, three, and four and so on from the current tag. Then we analyze what content we can enter so that it can reduce the distance between 


\section{Questions Answered : Light}

Cause Statement :Light is a form of energy and possess the properties of both waves and particles.

Effect Statement : (1) Light is a form of electromagnetic radiation of a certain wavelength or frequency. (2) These wavelength is easily visible to the naked eye of the living creatures. (3) Photons are the tiny fundamental energy packets of light. (4) The speed of the light in vacuum is $299,792,458 \mathrm{~m} / \mathrm{s}$. (5) Light travels in a linear motion and when it gets obstructed by an opaque object, the shadow is formed.

\section{Summary Reference Tags Question Raised}

Add to Cart

Figure 3: Student enters the content for Light

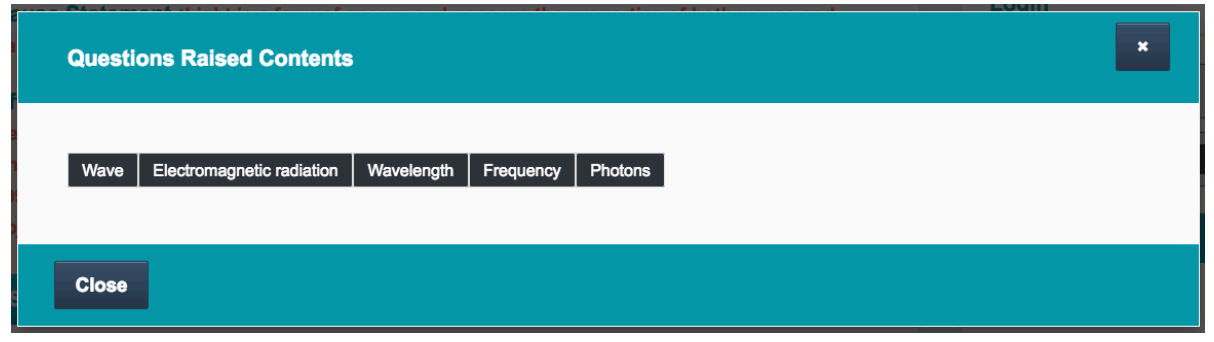

Figure 4: Tags for Light: Wave, Electromagnetic radiation, Wavelength, Frequency, Photons. 


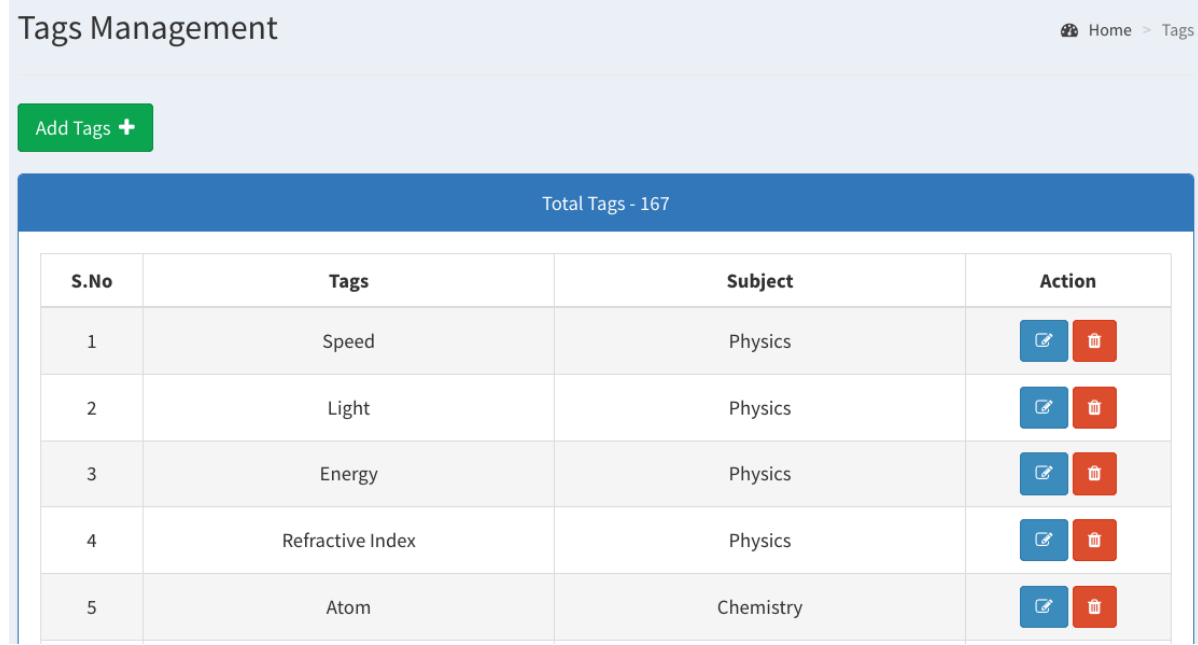

Figure 5: This is backend entry of tags

\section{Add Tags}

\section{EAdd Tags}

Tag Name :

Enter Tag Name

Tag Subject :

-- Select Subject --

\section{Submit}

Cancel

Figure 6: Tags can be entered by this Add tags feature 


\begin{tabular}{|c|c|c|}
\hline All Questions Management & \\
\hline & Total Questions - 167 & \\
\hline S.No & All Questions & Subject \\
\hline 1 & Speed & Physics \\
\hline 2 & Light & Physics \\
\hline 3 & Energy & Physics \\
\hline 4 & Refractive Index & Physics \\
\hline 5 & Atom & Chemistry \\
\hline 6 & Mass & Chemistry \\
\hline 7 & Quantum number & Chemistry \\
\hline
\end{tabular}

Figure 7: All the tags are automatically add to the Questions table

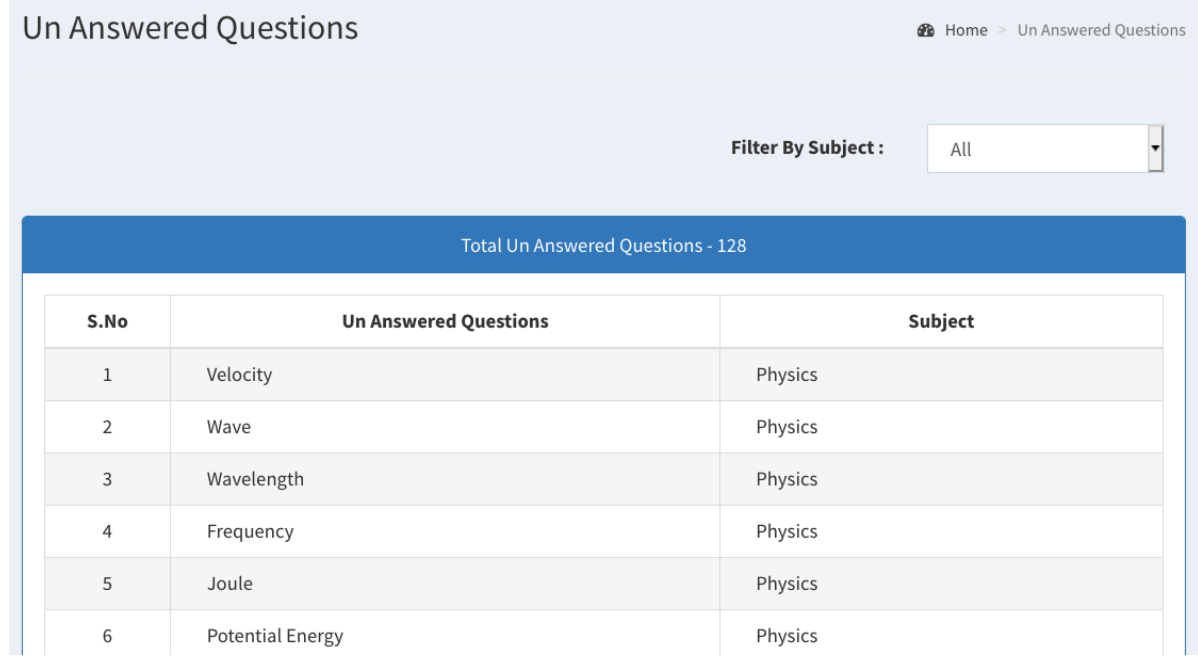

Figure 8: The questions which are not having any corresponding answers are entered in the unanswered questions table. Students can pick up topics from this table to search for answers to these questions. 


\section{Contents Management}

\section{Add Contents +}

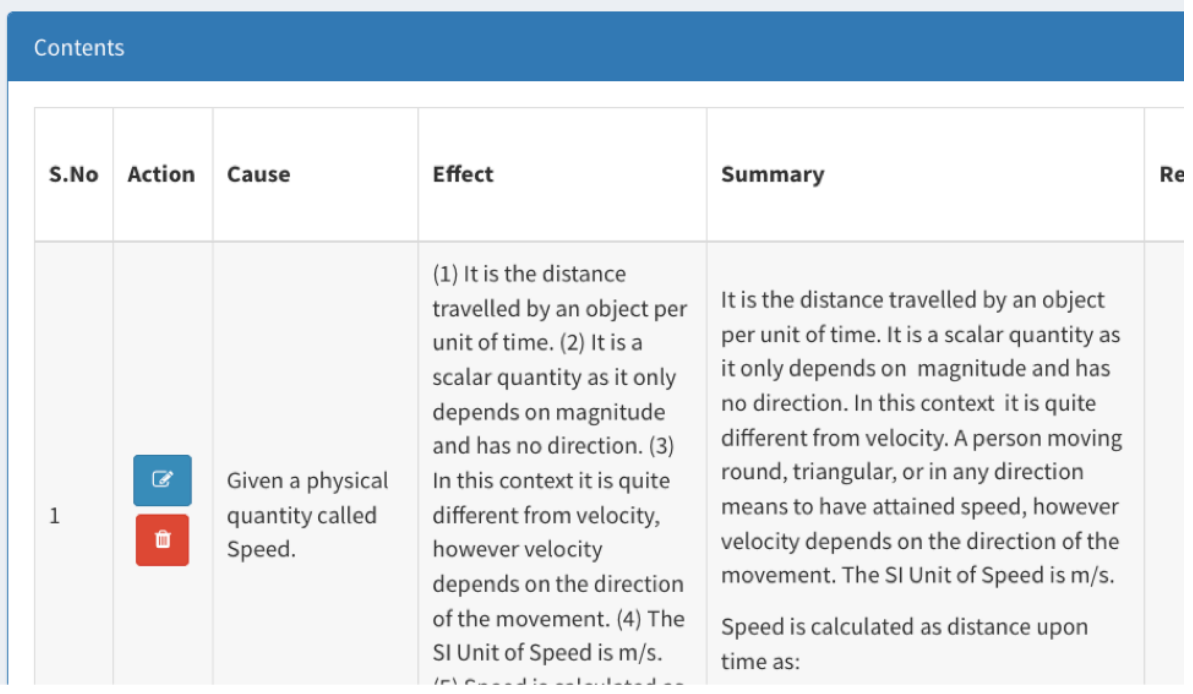

Figure 9: Contents are entered in the backend

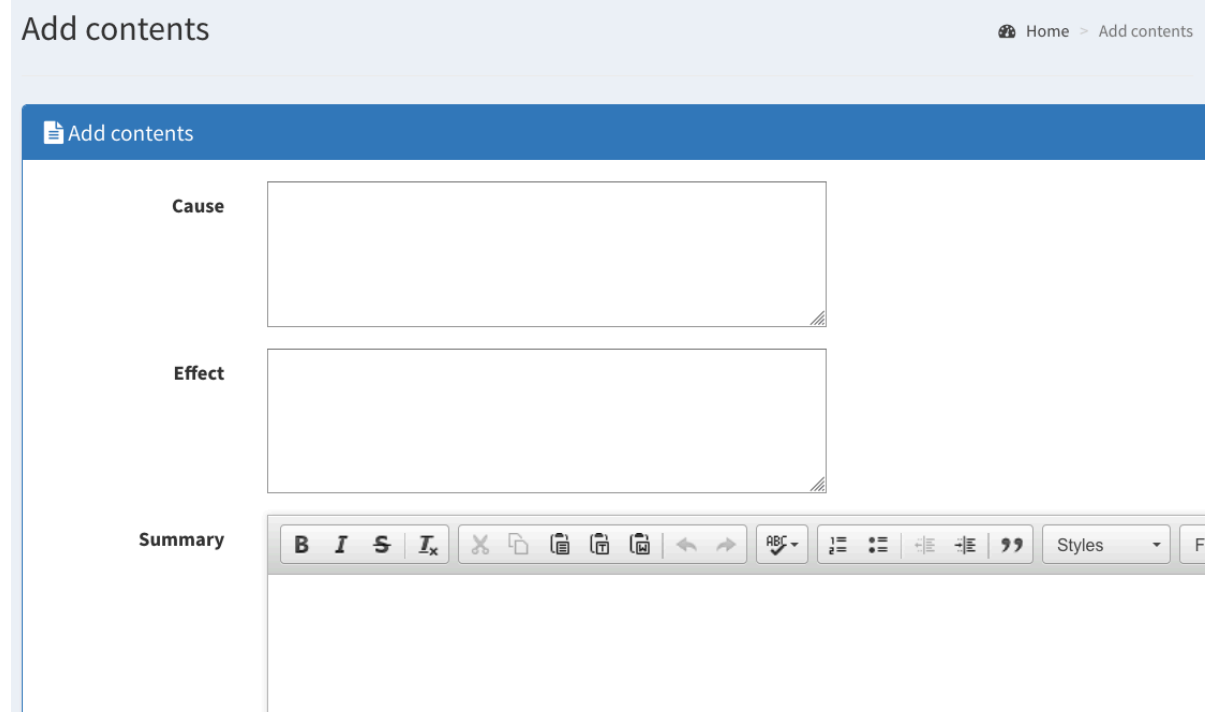

Figure 10: Contents entry 


\section{Effect Tags}

\begin{tabular}{c|l|} 
Name Entity & Newton's First law $x$ Velocity $x$ \\
Property & Select Some Options \\
Relation & Select Some Options \\
Covariates & Select Some Options \\
Questions answered & Speed $x$ Cancel \\
Sequence & Select Some Options \\
\hline & \\
\hline
\end{tabular}

Figure 11: Content entry with tags and Question answered, this contents answers the content for speed, with corresponding tags - Newtons first law and velocity

two tags.

Also since we have Graph of terminologies, if one person searches for a terminology then we can even report back the content for related terminology. For example, if user searches for "speed", it gives content for speed, but speed has a tag "Newtons First Law" and Newtons First Law has a tag "inertia". So a search for "speed" will give content for "Newtons First Law" and "inertia" also.

And all this we are able to do in a very cheap manner in a three thousand dollar project.

\section{Conclusion}

Conclusion. We have proposed a very cheap alternative to the content development, where we add the content that reduces the distance between the terminologies. It also linearizes the process of Literature search for a student. And for the person who is searching the database for the content, it searches the 
database in the form of nodes, and gives results for nodes that are at one, two

or three distance from the current node.

Since this project doesnt use any AI, it enables researchers or students to be in total control of the working methodology.

It is said that the more brains one uses, lesser is the material one requires. This statement is well shown by our project. The complicated tasks that Google is doing by spending billions of dollars, we have demonstrated that process in three thousand dollars.

\section{References}

[1] C. Haythornthwaite, Social network analysis: An approach and technique for the study of information exchange, Library Information Science Research 18 (4) (1996) 323 - 342. doi:https://doi.org/10.1016/S0740-8188(96) 90003-1.

[2] M. Steketee, A. Miyaoka, M. Spiegelman, Social network analysis, in: J. D. Wright (Ed.), International Encyclopedia of the Social Behavioral Sciences (Second Edition), second edition Edition, Elsevier, Oxford, 2015, pp. 461 467. doi:https://doi.org/10.1016/B978-0-08-097086-8.10563-X.

[3] D. L. Hansen, B. Shneiderman, M. A. Smith, Chapter 3 - social network analysis: Measuring, mapping, and modeling collections of connections, in: D. L. Hansen, B. Shneiderman, M. A. Smith (Eds.), Analyzing Social Media Networks with NodeXL, Morgan Kaufmann, Boston, 2011, pp. 31 - 50. doi:https://doi.org/10.1016/B978-0-12-382229-1.00003-5.

[4] J. K. Maldonado, Chapter 17 - the practical and policy relevance of social network analysis for disaster response, recovery, and adaptation, in: E. C. Jones, A. Faas (Eds.), Social Network Analysis of Disaster Response, Recovery, and Adaptation, Butterworth-Heinemann, 2017, pp. 255 - 267. doi:https://doi.org/10.1016/B978-0-12-805196-2.00017-0. 
[5] N. Kapucu, F. Demiroz, Chapter 3 - interorganizational networks in disaster management, in: E. C. Jones, A. Faas (Eds.), Social Network Analysis of Disaster Response, Recovery, and Adaptation, Butterworth-Heinemann, 2017, pp. 25 - 39. doi:https://doi.org/10.1016/B978-0-12-805196-2. $00003-0$.

[6] D. M. Varda, Chapter 4 - strategies for researching social networks in disaster response, recovery, and mitigation, in: E. C. Jones, A. Faas (Eds.), Social Network Analysis of Disaster Response, Recovery, and Adaptation, Butterworth-Heinemann, 2017, pp. 41 - 56. doi:https://doi.org/10. 1016/B978-0-12-805196-2.00004-2.

[7] J. Lu, Chapter 10 - interorganizational network dynamics in the wenchuan earthquake recovery, in: E. C. Jones, A. Faas (Eds.), Social Network Analysis of Disaster Response, Recovery, and Adaptation, ButterworthHeinemann, 2017, pp. 143 - 159. doi:https://doi.org/10.1016/ B.978-()-12.-805196-2.0001(0-8.

[8] A. Faas, E. C. Jones, Chapter 2 - social network analysis focused on individuals facing hazards and disasters, in: E. C. Jones, A. Faas (Eds.), Social Network Analysis of Disaster Response, Recovery, and Adaptation, Butterworth-Heinemann, 2017, pp. 11 - 23. doi:https://doi.org/10. 1016/B978-0-12-805196-2.00002-9.

[9] U. Brandes, Social network algorithms and software, in: J. D. Wright (Ed.), International Encyclopedia of the Social Behavioral Sciences (Second Edition), second edition Edition, Elsevier, Oxford, 2015, pp. $454-460$. doi:https://doi.org/10.1016/B978-0-08-097086-8.43121-1. 